\title{
EFEITO DO EXERCÍCIO PRÉVIO NO CICLISMO DE CURTA DURAÇÃO
}

\author{
EFFECT OF PRIOR EXERCISE ON SHORT-DURATION CYCLING
}

EFECTO DEL EJERCICIO PREVIO SOBRE EN EL CICLISMO DE CORTA DURACIÓN

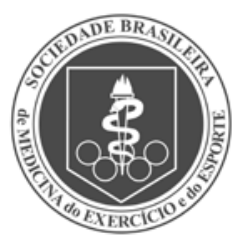

Artigo Original
Renato Aparecido Corrêa Caritá' (Educador Físico)

Fabrizio Caputo² (Educador Físico)

Camila Coelho Greco'

(Educadora Física)

Benedito Sérgio Denadai

(Educador Físico)

\section{Laboratório de Avaliação da Performance Humana da Universidade Estadual Paulista "Júlio de Mesquita Filho" (Unesp) Campus Rio Claro, Rio Claro, SP, Brasil. \\ 2. Laboratório de Pesquisas em Desempenho Humano, Centro de Ciências da Saúde e do Esporte da Universidade do Estado de Santa Catarina. (UDESC), Florianópolis, SC, Brasil.}

\section{Correspondência:}

Camila Coelho Greco. Laboratório de Avaliação da Performance Humana, UNESP, Avenida 24A, 1.515, Bela Vista; 13506-900, Rio Claro, SP, Brasil. grecocc@rc.unesp.br

\section{RESUMO}

Introdução: O exercício prévio tem importantes implicações na preparação de atletas antes de competições. Objetivo: Analisar o efeito de um exercício prévio realizado no domínio pesado no pico de torque $\left(P_{\text {TORQue }}\right)$ medido após exercício severo. Métodos: Participaram deste estudo 14 homens ativos (idade: $26 \pm 4$ anos, $\mathrm{VO}_{2 \text { max }}: 44 \pm 6 \mathrm{mLO}_{2} \cdot \mathrm{min}^{-1} \cdot \mathrm{kg}^{-1}$ ) que realizaram sete testes em dias diferentes: a) teste progressivo de rampa para determinação do $\mathrm{VO}_{2 \max }$ e da potência pico; b) quatro testes de carga constante para determinação da potência crítica, capacidade de trabalho anaeróbio e potência correspondente ao tempo de exaustão de 3 min $\left(P_{\text {TLim3min }}\right)$ e; $c$ ) dois testes de carga constante de 2 min na $P_{\text {TLim3min }}$ seguidos por um sprint all out de $10 \mathrm{~s}$, a fim de medir o $\mathrm{P}_{\text {TORouE. }}$ Este último protocolo foi realizado com (EP) e sem (CON) a realização de um exercício prévio pesado. Resultados: $O P_{\text {ToRQuE }}$ foi significantemente maior após o EP $(101 \pm 30 \mathrm{Nm})$ em relação à condição $\mathrm{CON}\left(95 \pm 23 \mathrm{Nm}\right.$ ). O tempo da resposta médio (TRM) do $\mathrm{VO}_{2}$ foi significantemente menor após o EP (24 \pm 7 s) em relação à condição $\mathrm{CON}\left(32 \pm 10\right.$ s). A amplitude primária do $\mathrm{VO}_{2}$ aumentou significantemente após o EP $\left(2598 \pm 421 \mathrm{mLO}_{2} \cdot \mathrm{min}^{-1}\right)$ em relação à condição CON $\left(2184 \pm 246 \mathrm{mLO}_{2} \cdot \mathrm{min}^{-1}\right)$. O déficit de $\mathrm{O}_{2}$ foi significantemente menor após o exercício prévio $\left(980 \pm 432 \mathrm{mLO}_{2}\right)$ em relação à condição CON $\left(1273 \pm 398 \mathrm{mLO}_{2}\right)$. Houve correlação significante entre a variação do déficit de $\mathrm{O}_{2}$ com a do $\mathrm{P}_{\text {TORQuE }}(r=0,53)$ e da variação do TRM com a do $P_{\text {TORQue }}(r=0,53)$. Conclusão: Pode-se concluir que o $P_{\text {TORQue }}$ é maior após exercício aeróbio de curta duração precedido do EP. Deste modo, esta estratégia pode ser interessante como preparação para algumas competições esportivas.

Palavras-chave: desempenho atlético, consumo de oxigênio, torque.

\section{ABSTRACT}

Introduction: Previous exercise has important implications in the preparation of athletes prior to competitions. Objective: To analyze the effect of previous heavy exercise on peak torque $\left(P_{\text {TORQuE }}\right)$ measured after severe exercise. Methods: Fourteen active males (age: $26 \pm 4$ years, $\mathrm{VO}_{2 \text { max: }} 44 \pm 6 \mathrm{mLO}_{2} \cdot \mathrm{min}^{-1} \cdot \mathrm{kg}^{-1}$ ) participated in this study and performed seven tests on different days: a) progressive ramp test for the determination of $\mathrm{VO}_{2 \max }$ and peak power; b) four constant workload tests to determine critical power, anaerobic work capacity and power corresponding to a time to exhaustion of $3 \mathrm{~min}\left(P_{\text {TLim3min }}\right)$, and; c) two constant workload tests of 2 min at $P_{\text {TLim3min }}$ followed by a

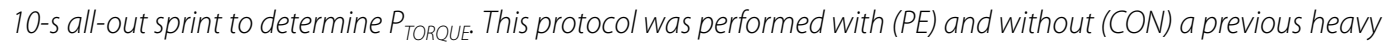
exercise. Results: The $P_{\text {TORQUE }}$ was significantly higher after the PE $(101 \pm 30 \mathrm{Nm})$ in relation to the CON condition $(95 \pm 23 \mathrm{Nm})$. The mean response time (MRT) of $\mathrm{VO}_{2}$ was significantly lower after PE $(24 \pm 7 \mathrm{~s})$ in relation to the $\mathrm{CON}$ condition ( $32 \pm 10 \mathrm{~s})$. The $\mathrm{VO}_{2}$ primary amplitude increased significantly after $P E\left(2598 \pm 421 \mathrm{mLO}_{2} \cdot \mathrm{min}^{-1}\right)$ in relation to the CON condition ( $\left.2184 \pm 246 \mathrm{mLO}_{2} \cdot \mathrm{min}^{-1}\right)$. The $\mathrm{O}_{2}$ deficit was significantly lower after PE $(980 \pm 432$ $\left.m L_{2}\right)$ in relation to the $\mathrm{CON}$ condition $\left(1273 \pm 398 \mathrm{~mL} \mathrm{O}_{2}\right)$. There was significant correlation between the variation of $\mathrm{O}_{2}$ deficit with that of $P_{\text {TORQUE }}(r=0.53)$ and the variation of MRT with that of $P_{\text {TORQUE }}(r=0.53)$. Conclusion: It can be concluded that the $P_{\text {TORQUE }}$ is higher after short-duration aerobic exercise preceded by heavy exercise. Therefore, this strategy may be interesting as preparation for some sport competitions.

Keywords: athletic performance, oxygen consumption, torque.

\section{RESUMEN}

Introducción: El ejercicio previo tiene importantes implicaciones en la preparación de atletas antes de competencias. Objetivo: Analizar el efecto de un ejercicio previo realizado en el dominio pesado en el pico de torque ( $\left.P_{\text {ToRquE }}\right)$ medido después de ejercicio severo. Métodos: Participaron en este estudio 14 hombres activos (edad: $26 \pm 4, \mathrm{VO}_{2}$ max: $44 \pm 6 \mathrm{mLO}_{2} \cdot \mathrm{min}^{-1} \cdot \mathrm{kg}^{-1}$ ) que realizaron 7 tests en días diferentes: a) test progresivo de rampa para determinación del $\mathrm{VO}_{2}$ max y de la potencia pico; b) 4 tests de carga constante para determinación de la potencia crítica, capacidad de trabajo anaeróbico y potencia correspondiente al tiempo de agotamiento de 3 min $\left.\left(P_{\text {TLim3min }}\right) y ; c\right) 2$ tests de carga constante de 2 min en la $P_{\text {TLim3min }}$ seguidos por un sprint all out de $10 \mathrm{~s}$, a fin de

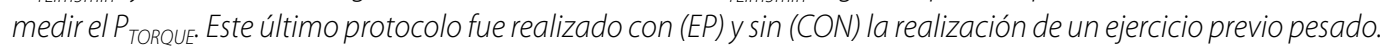
Resultados: El P TORQuE fue significativamente mayor después del EP (101 \pm 30 Nm) en relación a la condición CON $\left(95 \pm 23 \mathrm{Nm}\right.$ ). El tiempo de la respuesta promedio (TRM) del $\mathrm{VO}_{2}$ fue significativamente menor después del EP 
(24 \pm 7 s) en relación a la condición CON (32 \pm 10 s). La amplitud primaria del $\mathrm{VO}_{2}$ aumentó significativamente después del EP $\left(2598 \pm 421 \mathrm{mLO}_{2} \cdot \mathrm{min}^{-1}\right)$ en relación a la condición CON $\left(2184 \pm 246 \mathrm{mLO}_{2} \cdot \mathrm{min}^{-1}\right)$. El déficit de $\mathrm{O}_{2}$ fue significativamente menor después del ejercicio previo $\left(980 \pm 432 \mathrm{mLO}_{2}\right.$ ) en relación a la condición CON (1273 $\pm 398 \mathrm{mLO}_{2}$ ). Hubo correlación significativa entre la variación del déficit de $\mathrm{O}_{2}$ con la del $P_{\text {TORQUE }}(r=0,53)$ y de la variación del TRM con la del $P_{\text {TORQUE }}(r=0,53)$. Conclusión: Se puede concluir que el $P_{\text {TORQuE }}$ es mayor después del ejercicio aeróbico de corta duración precedido del EP. De este modo, esta estrategia puede ser interesante como preparación para algunas competiciones deportivas.

Palabras clave: rendimiento atlético, consumo de oxígeno, torque.

\section{INTRODUÇÃO}

O aquecimento tem sido considerado como um importante aspecto da preparação de atletas antes da realização das provas competitivas. Entre os aspectos importantes do aquecimento estão o aumento do metabolismo, da temperatura corporal e da ativação neuromuscular. Estudos na literatura têm mostrado que a performance aeróbia de curta duração (2-5 min) pode ser aumentada com a realização de um exercício prévio ${ }^{1,2}$. No entanto, parece haver uma relação ótima entre a intensidade do exercício prévio e a duração da recuperação, para que a tolerância ao exercício subsequente possa ser aumentada. Esta combinação provavelmente otimiza o balanço entre os efeitos positivos do exercício prévio na cinética do consumo de oxigênio $\left(\mathrm{VO}_{2}\right)$ e o tempo suficiente de recuperação da homeostase muscular (restauração de creatina fosfato e íons $\mathrm{H}^{+}$).

Durante o exercício realizado no domínio severo [i.e., acima da potência crítica (PC) - assíntota da relação potência-tempo], a resposta do $\mathrm{VO}_{2}$ e as reservas anaeróbias de energia [capacidade de trabalho anaeróbio ( $\left.W^{\prime}\right)$ - grau de curvatura da relação potência-tempo] têm sido consideradas aspectos importantes para a tolerância ao exercício $^{3}$. De fato, no domínio severo a exaustão tende a estar associada à obtenção do consumo máximo de oxigênio e ao esgotamento das reservas anaeróbias de energia4-6. Estudos têm mostrado que o exercício prévio pode acelerar a resposta do $\mathrm{VO}_{2}$ no início do exercício e reduzir o déficit de oxigênio ${ }^{7}$, aumentar a amplitude da resposta do $\mathrm{VO}_{2} 6,8$ aumentar a tolerância ao exercício (i.e., tempo limite - tlim) ${ }^{9}$ e a performance (i.e., potência média) no exercício severo subsequente ${ }^{1}$, pois diminuiria a utilização das reservas anaeróbias e retardaria o esgotamento das mesmas.

A W' representa a máxima quantidade de trabalho que pode ser realizada acima da $P C$ independentemente da sua taxa de utilização. Inicialmente, foi postulado que a W' reflete a reserva finita de energia composta por fosfagênios, o componente anaeróbio glicolítico e o estoque de $\mathrm{O}_{2}{ }^{10}$. Mais recentemente, a $W^{\prime}$ tem sido proposta para representar a capacidade de trabalho mecânico que pode ser usada enquanto que alguns fatores intramusculares relacionados à fadiga, como o pH e a concentração de fosfocreatina, declinam a um limite tolerável crítico $^{3}$. A fadiga tem sido definida como a redução da capacidade de produzir força ${ }^{11}$. Entretanto, as evidências que sustentam a possível relação entre a fadiga e os estoques de energia anaeróbia (i.e., W') ainda não foram apropriadamente demonstradas. Portanto, o objetivo deste estudo foi analisar o efeito de um exercício prévio realizado no domínio pesado na força muscular medida após exercício severo. Isto foi realizado analisando o pico de torque ( $\left.P_{\text {TORQUE }}\right)$ aplicado no pedal durante um exercício de máxima intensidade (i.e., all-out) subsequente a um exercício severo durante $2 \mathrm{~min}$. Baseado nos estudos de Jones et al. ${ }^{3}$ e Bailey et $a l^{5}{ }^{5}$, foi hipotetizado que o pico de torque será maior após o exercício severo que é precedido por um exercício pesado.
O objetivo deste estudo foi analisar o efeito de um exercício prévio realizado no domínio pesado no $\mathrm{P}_{\text {TORQUE }}$ medido após exercício severo.

\section{MATERIAL E MÉTODOS}

Quatorze indivíduos ativos do sexo masculino (idade: $26 \pm 4$ anos, estatura: $177 \pm 6 \mathrm{~cm}$, massa corporal: $78 \pm 7 \mathrm{Kg}$ ) participaram deste estudo. $\mathrm{O}$ estudo foi aprovado pelo Comitê de Ética da Instituição. Todos os indivíduos assinaram um termo de consentimento livre e esclarecido antes do início do protocolo experimental, elucidando todos os procedimentos e os possíveis riscos e benefícios associados aos mesmos.

Os indivíduos visitaram o laboratório em sete ocasiões durante um período de 3 semanas, sendo cada visita separada por 24 - $48 \mathrm{~h}$. Após completar o teste progressivo de rampa (visita 1), foram realizados quatro testes de carga constante (visitas 2 a 5) para estimar a PC e a W'. Posteriormente, todos os indivíduos completaram em ordem randômica 2 testes de curta duração, com e sem a realização do $E P$, no qual ao final o pico de torque $\left(P_{\text {TORQue }}\right)$ foi mensurado. Os indivíduos foram instruídos a chegarem ao laboratório hidratados, alimentados, sem realizar ingestão de cafeína ou álcool e sem realizar esforços extenuantes 24 horas antes dos testes. Todos os testes foram realizados no mesmo horário ( $\pm 2 \mathrm{~h}$ ) e com temperatura do ar controlada em $19-21^{\circ} \mathrm{C}$

\section{Protocolo progressivo de rampa}

Na primeira visita ao laboratório os indivíduos realizaram testes incrementais para determinação do limiar de lactato (LL), do $\mathrm{VO}_{2 \max }$ e potência pico $\left(P_{\text {PICO }}\right)$. Todos os testes foram realizados em um cicloergômetro de frenagem eletromagnética (Lode Excalibur Sport, Groningen, Holanda). Inicialmente os indivíduos pedalaram cinco estágios com incrementos de 25 W a cada $3 \mathrm{~min}$. Ao final de cada estágio foi realizada uma coleta de sangue de $25 \mu \mathrm{L}$ do lóbulo da orelha, que foram acondicionados em tubos Eppendorf contendo $50 \mu \mathrm{L} \mathrm{NaF}$ (1\%), para dosagem da concentração de lactato ([La]) (YSL, 2300 STAT, Yellow Springs, Ohio, EUA). A frequência de pedalada foi mantida entre 70 - 90 rpm. Esses estágios prévios foram realizados para determinação do $L^{12}$. Posteriormente, os voluntários descansaram por 30 min e iniciaram o teste progressivo de rampa pedalando 4 min a 90\% LL e seguido aumento de $25 \mathrm{~W} \cdot \mathrm{min}^{-1}$, com frequência de pedalada mantida entre 70 - 90 rpm até a exaustão voluntária. O ajuste individual (posição do guidão e selim) foi registrado e mantido em todos os testes. $\mathrm{O} \mathrm{VO}_{2}$ foi mensurado respiração a respiração durante todo o teste, a partir do gás expirado (QuarkPFTergo, Cosmed, Itália), sendo os dados suavizados e obtidas as médias de $15 \mathrm{~s}$. A calibração do sistema de análise das concentrações de $\mathrm{O}_{2}$ e $\mathrm{CO}_{2}$ foi realizada antes de cada teste, usando ar ambiente e um gás com concentrações conhecidas de $\mathrm{O}_{2}$ e $\mathrm{CO}_{2}$ de acordo com as instruções do fabricante. A turbina do analisador foi calibrada através de uma seringa de três litros. $\mathrm{O}$ mais alto $\mathrm{VO}_{2}$ obtido durante $15 \mathrm{~s}$ foi considerado como o $\mathrm{VO}_{2 \max }$ Todos os sujeitos preencheram no mínimo 
dois dos três critérios para o $\mathrm{VO}_{2 \max }: 1$ ) razão de trocas gasosas $(\mathrm{R})>1,1$; 2) [La] pico maior que $8 \mathrm{mM}, \mathrm{e} ; 3)$ frequência cardíaca máxima $\left(\mathrm{FC}_{\max }\right)$ no mínimo igual $90 \%$ da máxima prevista para idade ${ }^{13}$. A $P_{\text {PICO }}$ foi considerada como a maior potência atingida durante o teste progressivo de rampa.

\section{Protocolo para determinação da PC e da W'}

Para estimar os parâmetros da PC e W', os indivíduos realizaram quatro testes (visitas 2 a 5) até a exaustão voluntária (tlim) nas intensidades de 75\%,80\%, 85\% e 100\% $\mathrm{P}_{\mathrm{PICO}}$ em ordem randômica. Antes de cada teste os indivíduos realizaram um aquecimento de 5 min a $100 \%$ LL e posteriormente descanso de 5 min. Os valores individuais de potência e tlim obtidos durante os testes de carga constante foram ajustados a partir dos três modelos de dois parâmetros $\left[P=W^{\prime} / t_{\text {lim }}\right)+$ $\left.C P ; t_{\text {lim }}=W^{\prime} /(P-P C) ; P=P C . t_{l i m}+W^{\prime}\right]$ para estimar a PC e a $W^{\prime 14,15}$. A PC e a $W^{\prime}$ determinadas pelas três equações foram comparadas e o modelo com o menor erro padrão da estimativa ${ }^{15,16}$ foi adotado. A estimativa da PC e da W' foi utilizada para determinar a intensidade do exercício prévio pesado (90\%PC) e a potência de tolerância em 3 min $\left(P_{\text {TLim3min }}\right)$ conforme equação $1^{15}$.

$$
P_{\text {TLim3min }}=\left(W^{\prime} / \text { Tlim }\right)+P C
$$

Onde: W': capacidade de trabalho anaeróbio; Tlim: tempo limite; PC: potência crítica.

\section{Protocolo do teste de curta duração}

As duas condições experimentais (visitas 6 e 7) foram realizados em ordem randômica, com 2 min de duração cada. Uma destas condições foi adotada como controle (CON - sem a realização do EP) e outra foi realizada após a execução do EP. Cada condição foi realizada por 2 min na carga $P_{\text {TLim3min, }}$ seguidos por um sprint all out (figura 1). Antes do início do sprint foi realizada contagem regressiva de 5 s e os voluntários foram instruídos para atingir o pico de potência tão rapidamente quanto possível e continuar a manter maximamente toda a duração do sprint (10 s). Não foi dado nenhum feedback de tempo aos sujeitos durante o sprint all out.

Na condição de curta duração com a realização do EP, os indivíduos desempenharam 6 min de EP pesado (90\%PC), com a cadência de pedalada mantida entre 70 - 90 rpm, seguidos por 7 min de recuperação no cicloergômetro.

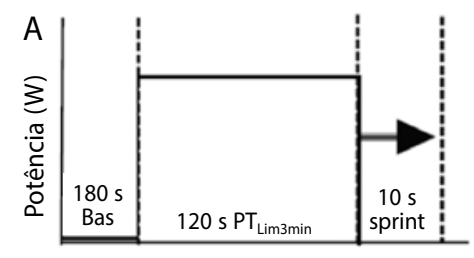

Tempo (s)

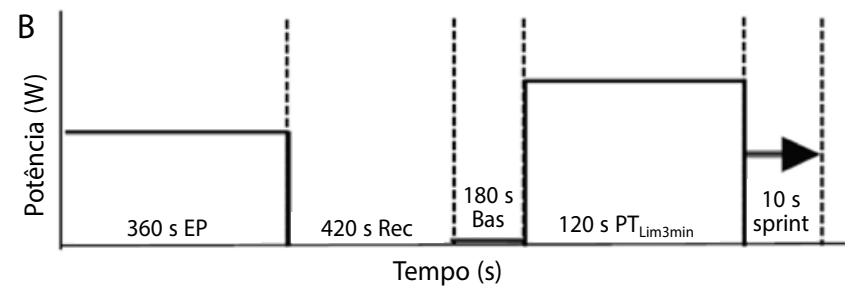

Figura 1. Esquema representativo do protocolo do exercício de curta duração. Acima (A) a condição controle, sem a realização do exercício prévio (EP), abaixo (B) a condição com a realização do EP.

\section{Protocolo para determinação do sprint all out}

O sprint all out foi estimado pelo fator linear conforme a equação 2, no qual foi calculada a potência de $50 \% \Delta\left(\mathrm{VO}_{2 \max }-\mathrm{LL}\right)$ e a média da cadência preferida adotada no teste progressivo de rampa.

Fator linear $=$ potência $(50 \% \Delta) /$ Cadência preferida²

Potência: carga ajustada em $50 \% \Delta\left(\mathrm{VO}_{2 \max }-\mathrm{LL}\right)$; cadência preferida 2 : cadência preferida ao quadrado.

Não houve intervalo entre o exercício de 2 min e o sprint all-out. Para garantir um esforço máximo no sprint all-out, os indivíduos foram instruídos a atingir o seu pico de potência tão rapidamente quanto possível, a partir do início do teste e para manter a cadência tão elevada quanto possível em todos os momentos do esforço, que durou $10 \mathrm{~s}$.

\section{Análise da cinética do $\mathrm{VO}_{2}$}

$\mathrm{O} \mathrm{VO}_{2}$ obtido respiração a respiração de cada condição de curta duração, sem e com a realização do EP, foi ajustado em relação ao tempo, no plano cartesiano. Em seguida, cada curva de resposta foi analisada manualmente para a exclusão de pontos extremos, que representam eventos fisiológicos não característicos da resposta do $\mathrm{VO}_{2}$ em exercício, que foram definidos como valores maiores que dois desvios-padrão da média local (4 - 5 respirações) ${ }^{17}$. Posteriormente, os dados foram interpolados para fornecer valores alinhados a cada segundo, para cada sujeito em sua transição ${ }^{8}$. A descrição matemática da cinética do $\mathrm{VO}_{2}$ foi realizada empregando-se o modelo mono-exponencial (Equação 3), sem tempo de atraso e com o janelamento do ajuste em $\mathrm{t}=0$ (equivalente ao tempo de resposta médio. TRM). A linha de base do $\mathrm{VO}_{2}$ foi definida como a média do $\mathrm{VO}_{2}$ medido durante os últimos $60 \mathrm{~s}$, mensurada previamente antes de cada condição.

$$
\mathrm{VO}_{2}(\mathrm{t})=\mathrm{VO}_{2 \mathrm{~b}}+\mathrm{A}\left[1-\mathrm{e}^{-(\mathrm{t} / \mathrm{\tau})}\right]
$$

Onde " $\mathrm{VO}_{2}$ " é o $\mathrm{VO}_{2}$ de base; "A" é a amplitude da resposta primária; "e" é o algarismo neperiano; " $\mathrm{t}$ " é o tempo do exercício; " $\tau$ " é a constante de tempo da elevação da resposta total.

O déficit de $\mathrm{O}_{2}\left(\mathrm{DO}_{2}\right)$ foi calculado de acordo com DiMenna et al. ${ }^{18}$, por meio da Equação 4:

$$
\mathrm{DO}_{2}\left(\mathrm{mLO}_{2}\right)=\mathrm{TRM} \cdot \Delta \mathrm{VO}_{2}
$$

Onde $\mathrm{DO}_{2}$ é o déficit de $\mathrm{O}_{2} ;$ TRM é o tempo de resposta médio e; $\Delta \mathrm{VO}_{2}$ é a diferença entre o $\mathrm{VO}_{2}$ e a amplitude da resposta primária.

\section{Análise do pico de torque}

O $\mathrm{P}_{\text {TORQUE }}$ foi calculado como sendo a média entre o maior valor obtido pela perna direita e esquerda mensurados durante o sprint all-out (10 s), calculados a partir das rotações executadas no ângulo de $360^{\circ}$. Os valores de torque, rotações e ângulos foram fornecidos pelo software do cicloergômetro Lode Excalibur Sport (Groningen, Holanda).

\section{Análise Estatística}

Os dados estão expressos como média \pm DP. A existência da normalidade dos dados foi verificada através do teste de Shapiro-Wilk. A análise obtida nas condições CON e EP foi realizada pelo teste $t$ de Student. A correlação entre as mudanças no TRM e no $\mathrm{DO}_{2} \mathrm{com}$ as mudanças no $\mathrm{P}_{\text {TORQue }}$ foi realizada pelo teste de correlação de Pearson. O nível de significância foi fixado em $p \leq 0,05$. Todas as análises estatísticas foram realizadas utilizando o programa estatístico SPSS 18.0. 


\section{RESULTADOS}

As variáveis máximas e submáximas obtidas no teste progressivo de rampa estão apresentadas na tabela 1.

A PC e a W' determinados em nosso estudo foram de $206 \pm 28 \mathrm{~W}$ e $19,4 \pm 4,5 \mathrm{Kj}$, respectivamente. O SEE e $\mathrm{r}^{2}$ foram $4 \pm 4 \mathrm{~W}$ e 0,98 $\pm 0,04$, respectivamente. Já a $P_{\text {TLim3min }}$ foi de $314 \pm 44$ W, representando 100,2 $\pm 5,1 \% \mathrm{P}_{\text {PICO }}$. O exercício prévio determinado em nosso estudo 90\%PC, foi realizado no domínio pesado de exercício (abaixo PC), sendo $184 \pm$ $26 \mathrm{~W}$ e representando $89 \pm 2 \%$ PC.

A [La] mensurada em repouso não foi diferente entre as condições CON e EP (1,2 \pm 0,3 e 1,3 \pm 0,2 mM, respectivamente). Já a [La] mensurada no final do exercício prévio e no final da recuperação foi de 4,6 \pm 1,2 mM e 2,9 \pm 0,6 mM, respectivamente. Não foram encontradas diferenças para a [La] e FC mensuradas no final do sprint all out entre as condições CON (10,6 $\pm 2,7 \mathrm{mM}$ e $179 \pm 10$ bpm, respectivamente) e EP (10,3 \pm 2 mM e $182 \pm 8$ bpm, respectivamente).

A tabela 2 mostra os valores das variáveis obtidas durante os testes de curta duração com e sem a realização do exercício prévio pesado. O TRM ajustou-se mais rapidamente após a realização do exercício prévio em relação à condição controle. A amplitude primária aumentou significantemente após a realização do exercício prévio em relação à condição controle. Já os valores de baseline e TD não foram significantemente diferentes. 0 déficit de $\mathrm{O}_{2}$ foi significantemente menor após a realização do exercício prévio em relação à condição controle $(p<0,05)$. A figura 2 mostra o ajuste do $\mathrm{VO}_{2}$ de um voluntário representativo durante o exercício de $2 \mathrm{~min}$.

$O P_{\text {TORQUE }}$ foi significantemente maior após a realização do EP em relação à condição $\operatorname{CON}(p<0,05)$. O $\mathrm{P}_{\text {TORQUE }}$ dos membros direito e esquerdo foi significantemente maior após a realização do EP em relação à condição $\operatorname{CON}(p<0,05)$. Não houve diferença significante no ângulo do $\mathrm{P}_{\text {TORQUe }}$ para os membros direito e esquerdo e para a frequência de pedalada pico ( $p>0,05$ ) (tabela 3). A figura 3 exemplifica os valores médios de $P_{\text {TORoue }}$ mensurados durante o sprint all out nas condições controle e com a realização do exercício prévio pesado de um voluntário representativo.

Houve correlação significante entre a variação do $\mathrm{DO}_{2}$ e a variação de $P_{\text {TORQue }}(r=0,53, p<0,05)$ e da variação do TRM com a variação do $P_{\text {TORQUE }}(r=0,53, p<0,05)$.

Tabela 1. Valores médios \pm DP das variáveis obtidas no protocolo incremental. $\mathrm{N}=14$.

\begin{tabular}{c|c}
\hline Variáveis & Média \pm DP \\
\hline $\mathrm{VO}_{2 \text { max }}\left(\mathrm{mLO}_{2} \cdot \mathrm{min}^{-1}\right)$ & $3446 \pm 425$ \\
\hline $\mathrm{P}_{\mathrm{PICO}}(\mathrm{W})$ & $314 \pm 40$ \\
\hline $\mathrm{FC}_{\max }(\mathrm{bpm})$ & $181 \pm 9$ \\
\hline$[\mathrm{La}]$ pico $(\mathrm{mM})$ & $9 \pm 3$ \\
\hline $\mathrm{LL}(\mathrm{W})$ & $96 \pm 34$
\end{tabular}

$\mathrm{VO}_{2 \text { max }}$ : consumo máximo de oxigênio; $\mathrm{P}_{\text {Pico: }}$ potência pico; $\mathrm{FC}_{\max }$ : frequência cardíaca máxima; [La]pico: concentração pico de lactato; LL: limiar de lactato.

Tabela 2. Valores médios \pm DP das variáveis obtidas no teste de curta duração, sem (CON) e com (EP) a realização do exercício prévio. $N=14$.

\begin{tabular}{c|c|c}
\hline Variáveis & CON & EP \\
\hline $\mathrm{VO}_{2 \mathrm{~b}}\left(\mathrm{mLO}_{2} \cdot \mathrm{min}^{-1}\right)$ & $1103 \pm 251$ & $1026 \pm 270$ \\
\hline $\mathrm{TRM}(\mathrm{s})$ & $32 \pm 10$ & $24 \pm 7^{*}$ \\
\hline $\mathrm{A}_{1}\left(\mathrm{mLO}_{2} \cdot \mathrm{min}^{-1}\right)$ & $2184 \pm 246$ & $2598 \pm 421^{*}$ \\
\hline $\mathrm{DO}_{2}\left(\mathrm{mLO}_{2}\right)$ & $1273 \pm 398$ & $980 \pm 432^{*}$ \\
\hline
\end{tabular}

$\mathrm{VO}_{2 b}$ : consumo de oxigênio de base; TRM: constante de tempo da elevação da resposta total; $A_{1}$ : amplitude da resposta total; $\mathrm{DO}_{2}$ : déficit de oxigênio. ${ }^{*} p<0,05$ em relação à condição $\mathrm{CON}$.

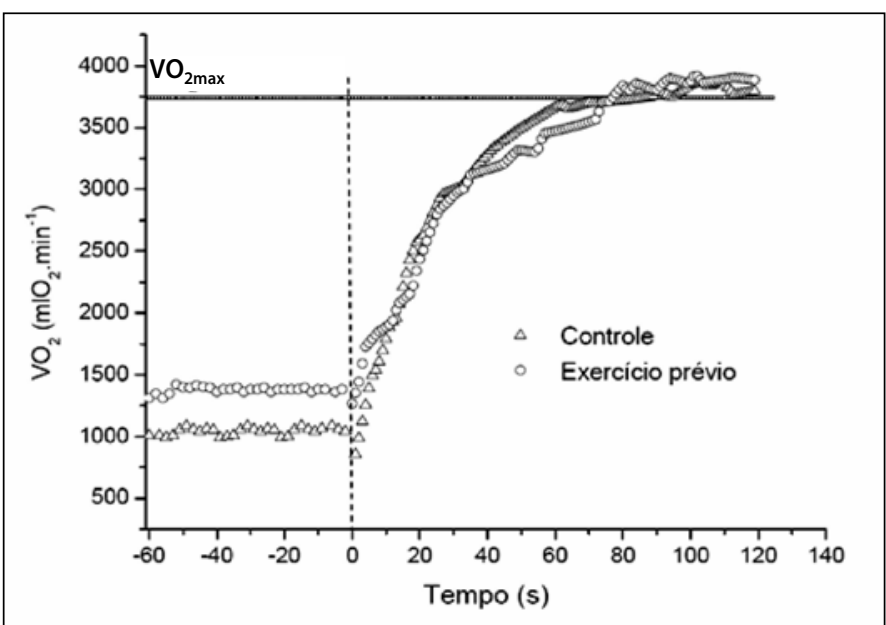

Figura 2. Valores interpolados de $\mathrm{VO}_{2}$ mensurados durante o exercício de 2 min com e sem a realização do exercício prévio pesado. Círculos abertos (com a realização do exercício prévio pesado - EP) e triângulos (sem a realização do exercício prévio pesado - $\mathrm{CON}$ ) representam o $\mathrm{VO}_{2}$ mensurado em um voluntário representativo.

Tabela 3. Valores médios \pm DP das variáveis obtidas no teste de sprint all-out sem (CON) e com (EP) a realização do exercício prévio. $\mathrm{N}=14$.

\begin{tabular}{c|c|c}
\hline Variáveis & CON & EP \\
\hline P $_{\text {TORQUE }}(\mathrm{Nm})$ & $95 \pm 23$ & $101 \pm 30^{*}$ \\
\hline Ângulo $\mathrm{P}_{\text {TORQUe }}$ (graus) & $88 \pm 9$ & $81 \pm 25$ \\
\hline Frequência de pedalada pico (RPM) & $112 \pm 9$ & $119 \pm 10$ \\
\hline
\end{tabular}

$\mathrm{P}_{\text {TORouE: }}$ pico de torque; Angulo $\mathrm{P}_{\text {TorouE: }}$ pico do ângulo de Torque; frequência de pedalada pico: pico da cadência atingida. ${ }^{*} p<0,05$ em relação à condição CON.

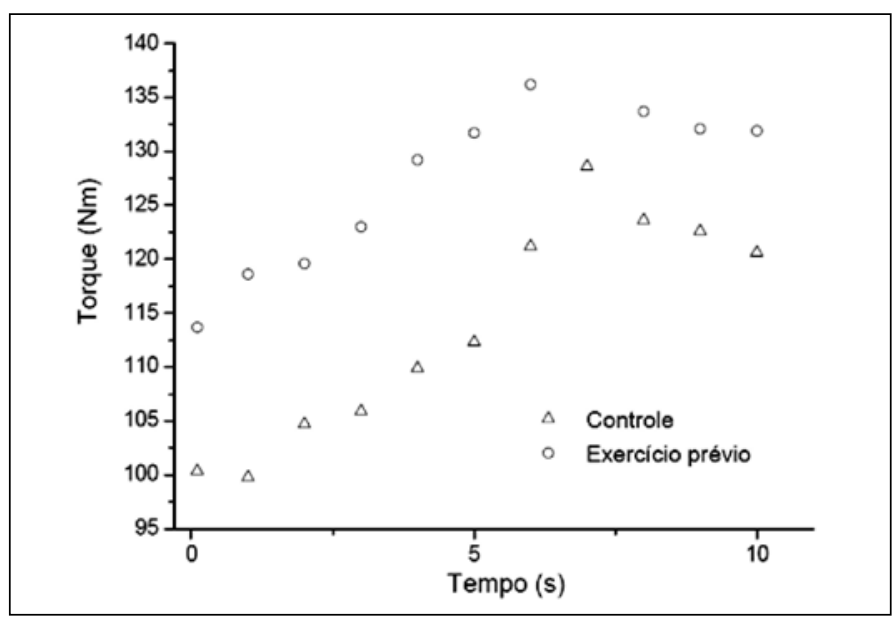

Figura 3. Valores de torque mensurados durante o sprint all out com (círculos) e sem (triângulos) a realização do exercício prévio pesado, em um voluntário representativo.

\section{DISCUSSÃO}

O principal e original achado deste estudo foi que o $\mathrm{P}_{\text {TORQUE }}$ durante o sprint all out foi maior com a realização do exercício prévio, o que confirma nossa hipótese inicial. Além disso, verificou-se uma cinética mais rápida do $\mathrm{VO}_{2}$ e consequentemente, um menor déficit de $\mathrm{O}_{2}$ após o EP, sendo estas mudanças correlacionadas com a mudança do $\mathrm{P}_{\text {TORQUE. }}$ Tomados em conjunto, estes dados confirmam e expandem a proposta de que a W' possa identificar a capacidade de trabalho mecânico que pode ser usada enquanto que alguns fatores intramusculares relacionados à fadiga, como o pH e a concentração de fosfocreatina, declinam a um limite tolerável crítico.

O primeiro aspecto que deve ser analisado neste estudo é a otimização da intensidade do EP e o período de recuperação anteriormente à sessão subsequente. A intensidade do EP adotada em nosso estudo (90\%PC) 
mostrou proporcionar uma perturbação no meio intracelular, causando um aumento na [La] no final do EP $(4,7 \pm 1,1 \mathrm{mM})$. Posteriormente, 0 tempo de recuperação utilizado entre as sessões permitiu remover parte desta [La] (2,9 \pm 0,6 mM). Jones et al. ${ }^{19}$ têm sugerido que [La] próximas a $3 \mathrm{mM}$ seriam potencialmente benéficas para a sessão subsequente de exercício. Por outro lado, estudos ${ }^{1,20}$ que verificaram uma [La] superior a 5 $\mathrm{mM}$ após a recuperação encontraram um efeito negativo na performance da sessão subsequente.

Jones et al. ${ }^{19}$ propõem que a acidose metabólica proporcionada pelo EP parece aumentar a disponibilidade de oxigênio para a sessão subsequente, determinando uma mudança para a direita da curva de dissociação da oxiemoglobina, causada pelo efeito de Bohr, no qual ocorre uma tendência do oxigênio deixar a corrente sanguínea quando a concentração de dióxido de carbono aumenta. Burnley et al. ${ }^{21}$ sugerem também que a maior atividade eletromiográfica muscular, que representa maior recrutamento de unidades motoras e a maior homogeneidade da perfusão muscular no início do exercício subsequente, também contribuem para a cinética de $\mathrm{VO}_{2}$ modificada. De fato, Saitoh et al. ${ }^{22}$ verificaram redução na heterogeneidade espacial da cinética de desoxigenação muscular no exercício pesado subsequente à um exercício pesado.

Os efeitos do EP sobre a tolerância ao exercício (tlim) têm sido amplamente estudados. Jones et al. ${ }^{7}$ analisaram o efeito do exercício pesado (i.e., $50 \%$ da diferença entre o limiar ventilatório e o $\mathrm{VO}_{2 \max }$ ) no tlim nas intensidades de 100, 110 e 120\% $V_{2 \max }$ em indivíduos ativos. Houve um aumento significante do tlim a 100\% (de 386 para 613 s), 110\% (de 218 para 284 s) e a $120 \% \mathrm{VO}_{2 \max }$ (de 139 para 180 s). Estes dados também foram encontrados em outros estudos ${ }^{1,19,21,23}$, nos quais a cinética de $\mathrm{VO}_{2}$ no início do exercício também foi acelerada. Este aumento da tolerância tem sido atribuído a menor utilização da W' no início do exercício subsequente, permitindo aumento do tempo de exaustão.

\section{REFERÊNCIAS}

1. Burnley M, Doust $J H$, Jones AM. Effects of prior warm-up regime on severe-intensity cycling performance. Med Sci Sports Exerc. 2005;37(5):838-45

2. Palmer CD, Jones AM, Kennedy GJ, Cotter JD. Effects of prior heavy exercise on energy supply and 4000-m cycling performance. Med Sci Sports Exerc. 2009;41(1):221-9.

3. Jones AM, Vanhatalo A, Burnley M, Morton RH, Poole DC. Critical power: implications for determination of $\mathrm{VO}_{2 \max }$ and exercise tolerance. Med Sci Sports Exerc. 2010;42(10):1876-90.

4. Coats EM, Rossiter HB, Day JR, Miura A, Fukuba Y, Whipp BJ. Intensity-dependent tolerance to exercise after attaining $\mathrm{VO}_{2 \max }$ in humans. J Appl Physiol. 2003;95(2):483-90.

5. Bailey SJ, Vanhatalo A, Wilkerson DP, Dimenna FJ, Jones AM. Optimizing the "priming" effect: influence of prior exercise intensity and recovery duration on $\mathrm{O}_{2}$ uptake kinetics and severe-intensity exercise tolerance. J Appl Physiol. 2009;107(6):1743-56.

6. Burnley, $\mathrm{M}$; Davison, $\mathrm{G}$; Baker, JR. Effects of priming exercise on $\mathrm{VO}_{2}$ kinetics and the power-duration relationship. Med Sci Sports Exerc 2011;43(11):2171-79.

7. Jones AM, Berger NJ, Wilkerson DP, Roberts CL. Effects of "priming" exercise on pulmonary $\mathrm{O}_{2}$ uptake and muscle deoxygenation kinetics during heavy-intensity cycle exercise in the supine and upright positions. J Appl Physiol. 2006;101(5):1432-41.

8. Burnley, M; Doust, JH; Carter, H; Jones, AM. Effects of prior exercise and recovery duration on oxygen uptake kinetics during heavy exercise in humans. Exp Physiol. 2001;86:417-25.

9. Carter H, Grice Y, Dekerle J, Brickley G, Hammond AJP, Pringle JSM. Effect of prior exercise above and below critical power on exercise to exhaustion. Med Sci Sports Exerc. 2005;37(5):775-81.

10. Fukuba Y, Miura A, Endo M, Kan A, Yanagawa K, Whipp BJ. The curvature constant parameter of the power-duration curve for varied-powerexercise. Med Sci Sports Exerc. 2003;35(8):1413-18.

11. GandeviaSC. Spinal and supraspinal factors in human muscle fatigue. Physiol Rev. 2001;81(4):1725-89.

12. Carter H, Jones AM, BarstowTJ, Burnley M, Williams CA, Doust JH. Oxygen uptake kinetics in treadmill running and cycle ergometry: a comparison. J Appl Physiol. 2000;89(3):899-907.
Segundo o nosso conhecimento, este foi o primeiro estudo que investigou os efeitos do EP sobre a fadiga após o exercício severo. A fadiga muscular tem sido definida como redução na força máxima exercida por um músculo ou grupo muscular devido a mecanismos centrais e/ou periféricos ${ }^{11}$. Em exercícios de curta duração (até 30 - 60 min), a origem da fadiga tende a ser predominantemente periférica, relacionada principalmente ao acúmulo de metabólitos (p.ex., fosfato inorgânico, lactato, íons hidrogênio, potássio e amônia). Recentemente, a W' tem sido proposta para refletir o acúmulo de metabolitos que induzem a fadiga (p.ex., acúmulo intramuscular de fosfato inorgânico e $\mathrm{H}^{+}$e acúmulo intersticial / extracelular de $\mathrm{K}^{+}$) para um limite tolerável crítico ${ }^{3}$. Verificamos em nosso estudo que o $\mathrm{P}_{\text {TORQUE }}$ após o exercício severo foi maior quando este foi precedido do EP. Além disso, houve correlação significante entre as mudanças no TRM $(r=0,53, p<0,05)$ e déficit do $\mathrm{O}_{2}(r=0,53, p<0,05)$ com as mudanças no $P_{\text {TORQue }}$ após EP. Nossos dados, portanto, suportam a utilização da $W^{\prime}$ como um indicador de acúmulo de metabólitos que induzem a fadiga.

\section{CONCLUSÃO}

Com base nestes resultados, pode-se concluir que o EP pesado pode proporcionar um efeito benéfico sobre a força muscular em exercícios de aeróbios de curta duração. Nossos dados e os existentes na literatura ${ }^{1,9,19}$ sugerem o uso desta estratégia (i.e., EP) para aumentar a tolerância e reduzir a fadiga em diferentes condições experimentais e de competição esportiva.

Todos os autores declararam não haver qualquer potencial conflito de interesses referente a este artigo.

13. Taylor HI, Buskirk E, Henschel A. Maximal oxygen intake as an objective measure of cardio-respiratory performance. J Appl Physiol. 1955;8(1):73-80.

14. Bull AJ, Housh TJ, Johnson GO, Perry SR. Effect of mathematical modeling on the estimation of critical power. Med Sci Sports Exerc. 2000;32(2):526-30.

15. Vanhatalo A, Jones AM, Burnley M. Application of Critical Power in Sport. International. J Sports Physiol Perform. 2011;6(1):128-36.

16. Hill DW, Smith JC. A method to ensure the accuracy of estimates of anaerobic capacity derived using the critical power concept. J Sport Med Phys Fitness. 1994;34(1):23-37.

17. Jones AM, Poole DC. Oxygen uptake dynamics: from muscle to mouth-an introduction to the symposium. Med Sci Sports Exerc. 2005;37(9):1542-50.

18. DiMenna FJ, Wilkerson DP, Burnley M, Jones AM. Influence of priming exercise on pulmonary $\mathrm{O}_{2}$ uptake kinetics during transitions to high-intensity exercise from an elevated baseline. J Appl Physiol. 2008;105(2):538-46

19. Jones AM, Koppo K, Burnley M. Effects of prior exercise on metabolic and gas exchange responses to exercise. Sports Med. 2003;33(13):949-71.

20. Wilkerson DP, Koppo K, Barstow TJ, Jones AM. Effect of work rate on the functional 'gain' of Phase II pulmonary $\mathrm{O}_{2}$ uptake response to exercise. Resp Physiol Neurob. 2004;142(2-3):211-23.

21. Burnley M, Doust JH, Jones AM. Effects of prior heavy exercise, prior sprint exercise and passive warming on oxygen uptake kinetics during heavy exercise in humans. Eur J Appl Physiol. 2002;87(4-5):424-32.

22. Saitoh T, Ferreira LF, Barstow TJ, Poole DC, Ooue A, Kondo N, Koga S. Effects of prior heavy exercise on heterogeneity of muscle deoxygenation kinetics during subsequent heavy exercise. Am J Physiol Regul Integr Comp Physiol. 2009;297(3):615-21.

23. Burnley M, Jones AM, Carter H, Doust JH. Effects of prior heavy exercise on phase II pulmonary oxygen uptake kinetics during heavy exercise. J Appl Physiol. 2000;89(4):1387-96. 